\title{
Analysis of the Tunable Asymmetric Fiber F-P Cavity for Fiber Strain Sensor Edge-Filter Demodulation
}

\begin{abstract}
Haotao CHEN and Youcheng LIANG*
Guangzhou Ivia Aviation College, Guangzhou, 510403, China

${ }^{*}$ Corresponding author: Youcheng LIANG

E-mail: liangyoucheng@caac.net

Abstract: An asymmetric fiber (Fabry-Pérot, F-P) interferometric cavity with the good linearity and wide dynamic range was successfully designed based on the optical thin film characteristic matrix theory; by adjusting the material of two different thin metallic layers, the asymmetric fiber F-P interferometric cavity was fabricated by depositing the multi-layer thin films on the optical fiber's end face. The asymmetric F-P cavity has the extensive potential application. In this paper, the demodulation method for the wavelength shift of the fiber Bragg grating (FBG) sensor based on the F-P cavity is demonstrated, and a theoretical formula is obtained. And the experimental results coincide well with the computational results obtained from the theoretical model.
\end{abstract}

Keywords: Fiber sensor, demodulation, asymmetric, F-P cavity, edge-filter

Citation: Haotao CHEN and Youcheng LIANG, "Analysis of the Tunable Asymmetric Fiber F-P Cavity for Fiber Strain Sensor Edge-Filter Demodulation," Photonic Sensors, 2014, 4(4): 338-343.

\section{Introduction}

Fiber optical sensors have been applied in various measurements because of their inherent advantages [1-3]. Fiber Bragg grating (FBG) sensors are the sensors in common use, which possess the high sensitivity, compact size, and survivability in harsh environments. FBG sensors have been widely used in fields such as temperature, strain, vibration, and displacement measurements [4]. For the high bandwidth application, it requires a practical demodulation method. At present, the main demodulation methods of FBG sensors include the Fourier transform method, linear edge-filter method, matching filter method, unbalanced Mach-Zender interferometer method, etc. The ratiometric wavelength monitor has the advantages of the simple configuration, high-speed measurement, and no mechanical movement [5]. For the cost-effective interrogation technique, linearly wavelength dependent devices based on various optical mechanisms, such as the Fabry-Pérot (F-P) filter [6], wavelength division multiplexing (WDM) coupler [7], long-period fiber gratings [8], and Sagnac loop filter [9], have been intensively developed, but these techniques still require more improvements in the stability, flexibility, and multi-point sensibility [10].

The linear edge filter method has the advantages of the simple structure and good practicality, and it is used widely in the FBG signal demodulation [11]. The most widely used fiber optical linear edge filter is the F-P filter.

The fiber F-P interferometric cavity which possesses the good sensitivity and resolution is widely used in the tunable filter, modulator, and fiber sensor. The basic F-P interferometer incorporates an in-line or internal reflector formed by the interface between the bond and fusion spliced fibers, the end face of the fiber typically having been pre-coated with a reflective dielectric layer such as

Received:12 December 2013/ Revised version: 29 August 2014

(C) The Author(s) 2014. This article is published with open access at Springerlink.com

DOI: $10.1007 / \mathrm{s} 13320-014-0158-3$

Article type: Regular 
titanium. Its transmittance or reflectance is a sine function [12]. It is well known that the linearity of sine function is not good and its range of linearity is very narrow, because around the maximum and the minimum, the responses are much slower than that far from the extreme. These limit the measurement range and sensitivity of the F-P interferometic fiber optical sensors. To design an F-P cavity optical sensor with the good linearity and wide dynamic range is what we long for.

The asymmetric F-P interferometer structure is an ideal project to resolve this problem, but it still has not been reported. In this paper, a tunable asymmetric F-P interferometer cavity with the good linearity and wide dynamic range is reported, and this F-P cavity is used as the edge-filter to demodulate the wavelength shift of the FBG strain sensor.

\section{Principles}

\subsection{Principle of asymmetric fiber F-P cavity}

The asymmetric F-P interferometric cavity, which consists of a dielectric tunable layer (usually air) between a high reflector considered as an ideal metal and a partial reflector consisting of two thin metallic films, is shown in Fig. $1 . S$ is the cavity length which is the distance between the two fusion spliced points on the micro capillary. Two single-mode optical fibers are cut perpendicular to the fiber axis with a fiber cleaver followed by a deposition of the high-reflectance coating in a vacuum evaporation chamber.

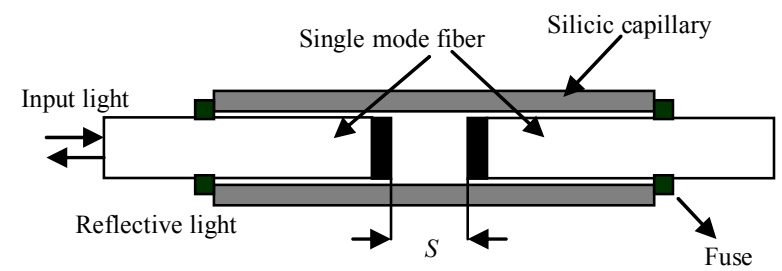

Fig. 1 Structure of the asymmetric optical fiber F-P cavity.
When the dielectric tunable layer of the F-P interferometer is very thin, this system can be considered as a multiple-layer thin film system and can be analyzed with the optical thin film theory. Here, the single-mode fiber (SMF) is considered as the incident medium; the metallic high reflector is considered as the substrate; a multiple-layer thin film consists of the middle dielectric layer and multiple thin metallic films which are deposited on the end face of the SMF. The thin film system can be expressed with the following formula:

$$
G\left|M_{1} M_{2} L\right| M_{g}
$$

where $G$ denotes the incident media, and its refractive index is written as $n_{0} . M_{1}$ and $M_{2}$ denote the metallic thin films deposited on the end face of the SMF; their complex refractive indices are written as $N_{1}$ and $N_{2}$, and the thicknesses are written as $d_{1}$ and $d_{2} . M_{g}$ denotes the substrate with the complex refractive index $N_{g}$. $L$ denotes the length of the dielectric tunable layer with the refractive index $n_{m}$ and the thickness $d_{m}$.

When the light is normal incidence, the interference matrix of the assembly can be written as

$$
\begin{aligned}
& {\left[\begin{array}{l}
B \\
C
\end{array}\right]=\left(\prod_{r=1}^{k}\left[\begin{array}{cc}
\cos \delta_{r} & \mathrm{j} \sin \delta_{r} / N_{r} \\
\mathrm{j} N_{r} \sin \delta_{r} & \cos \delta_{r}
\end{array}\right]\right) \times} \\
& {\left[\begin{array}{cc}
\cos \delta_{m} & \mathrm{j} \sin \delta_{m} / n_{m} \\
\mathrm{j} n_{m} \sin \delta_{m} & \cos \delta_{m}
\end{array}\right]\left[\begin{array}{c}
1 \\
N_{g}
\end{array}\right]}
\end{aligned}
$$

where $\delta_{r}=2 \pi N_{r} d_{r} / \lambda(r=1,2), \delta_{m}=2 \pi n_{m} d_{m} / \lambda$, and $\delta$ denotes the phase thickness.

And the reflectance $R$ of the assembly is given as follows:

$$
R=\left|\frac{n_{0}-\frac{C}{B}}{n_{0}+\frac{C}{B}}\right|^{2} .
$$

The reflectance is given as

$$
R=1-\frac{16 \pi n_{0} k_{1}^{2} d_{1} c_{1}\left(c_{1} \lambda-2 \pi d_{1} c_{2}-2 \pi d_{2} c_{2}\right)}{\left[n_{0}\left(c_{1} \lambda-2 \pi d_{1} c_{2}-2 \pi d_{2} c_{2}\right)+4 \pi k_{1}^{2} d_{1} c_{1}\right]^{2}+\left(2 \pi k_{2}^{2} d_{2} c_{1}-c_{2} \lambda\right)^{2}}
$$


where $c_{1}=\cos \delta_{m}+\frac{k_{g} \sin \delta_{m}}{n_{m}}, c_{2}=n_{m} \sin \delta_{m}-k_{g} \cos \delta_{m}$.

The influence of various parameters on the reflectivity of the film can be analyzed by (3), and the best response curve of the cavity can be obtained. In this paper, the parameters of the material for the cavity are shown as follows: $n_{0}=1.45$ (fiber), $N_{g}=$ 0.2-6.27j (copper layer, $\mathrm{Cu}$ ), $n_{m}=1$ (air layer), wavelength $\lambda=1550$ (nanometer, $\mathrm{nm}$ ), $N_{1}=$ $3.5-3.5 \mathrm{j}, d_{1}=6 \mathrm{~nm}$ (chromium, $\mathrm{Cr}$ ), $N_{2}=0.2-6.27 \mathrm{j}$, $d_{2}=12 \mathrm{~nm}(\mathrm{Cu})$.

Figure 2 shows the calculated reflectivity response curve of the F-P cavity with the cavity length of $60.2 \mu \mathrm{m}$. From Fig. 2, we can see that the descending and ascending intervals of the cavity with the fixed cavity length have the good linearity. The monotony ascending interval has been compressed. At the same time, the monotony descending interval is close to $\pi$, so the dynamic range of the interferometric cavity can be enlarged evidently.

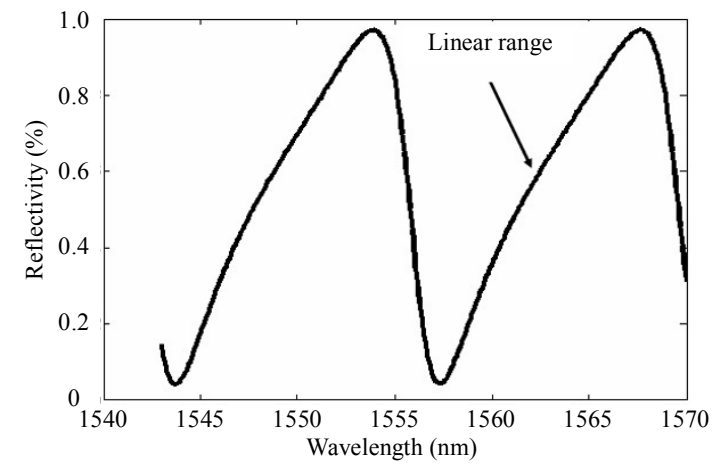

Fig. 2 Calculated reflectivity response curve of the asymmetric F-P cavity.

Because the up interval of the reflection curve between the trough and crest is nearly linear, this fiber F-P cavity can be used as a linear edge filter demodulating the FBG wavelength shift. Like the basic F-P cavity, the cavity length of the asymmetric F-P cavity determines the spectral interference curve length. And the free spectral space can be obtained by adjusting the cavity length. When the length of the F-P cavity is constant, the linear range of the reflectance curve between the trough and crest can be approximated to

$$
R=A \lambda+B
$$

where $A$ is the slope of the linear filter, and $B$ is the ordinate at the origin of the straight line.

\subsection{Principle of demodulation system}

The demodulation setup is shown in Fig. 3. The input light from the broadband light source (BBS) is introduced into the FBG sensor by the first 3-dB coupler. And the narrow band spectrum reflected by the FBG sensor is split into two beams by the other $3-\mathrm{dB}$ coupler. One of the optical beams is linear filter reflected by the F-P filter and reaches the photoelectric detector through the 3-dB coupler. Another beam is directly detected to compensate the effect of intensity fluctuation of the light source on the experimental result. The optical signals detected by the two detectors are amplified by the amplifiers and then are output to the divider for the data processing.

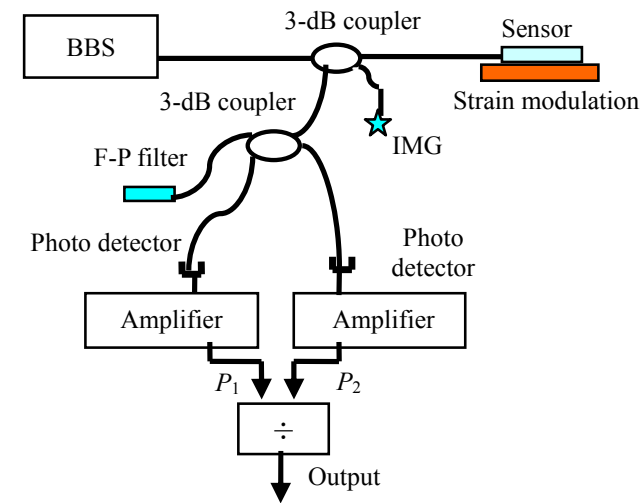

Fig. 3 Scheme of the F-P edge filter demodulation system.

As shown in Fig. 3, $P_{1}(\lambda)$ is the reflective optical power through the linear filter (signal light), and $P_{2}(\lambda)$ is the optical power detected by the detector directly (reference light).

$$
P_{1}(\lambda)=\int_{-\infty}^{+\infty} F\left(\lambda-\lambda^{\prime}\right) R\left(\lambda^{\prime}\right) d \lambda^{\prime}
$$

where $F(\lambda)$ is the reflected light power spectral density, and $R(\lambda)$ is the transfer function of the edge-filter.

In the linear wavelength range of a linear filter, $R(\lambda)$ is approximated to a linear function of $\lambda$, and the spectral width of $F(\lambda)$ is much smaller than the 
linear wavelength range. So the detected optical power $P_{1}(\lambda)$ can also be approximated to a linear function of $\lambda$. And $P_{1}(\lambda)$ can be written as

$$
P_{1}(\lambda)=R(\lambda) \int_{-\infty}^{+\infty} F\left(\lambda-\lambda^{\prime}\right) d \lambda^{\prime}
$$

where $\int_{-\infty}^{+\infty} F\left(\lambda-\lambda^{\prime}\right) d \lambda^{\prime}=P_{2}(\lambda)$

The transfer function of the fiber F-P edge-filter cavity can be given as

$$
R(\lambda)=\frac{P_{1}(\lambda)}{P_{2}(\lambda)}
$$

From (6) and (7), we can find that the wavelength shift of the FBG sensor $\Delta \lambda$ is a linear function of $P_{1}(\lambda) / P_{2}(\lambda)$. And the wavelength information of the sensing sensor can be obtained by testing the value of $P_{1}(\lambda) / P_{2}(\lambda)$ which provides an edge-filter linear method for us to demodulate the transmission signal of FBG sensor.

\section{Experimental results and discussion}

Figure 4 shows the reflectivity curve of the asymmetric fiber F-P cavity. The structural parameters of the F-P cavity are matched with the data as previously described. From Figs. 2 and 4, we can find that the descending interval of the reflectivity curve is enlarged. Simultaneously, the linearity of the response is improved in the monotony interval. But it appears that there are some deviations between the experimentally measured reflectivity of the minimum and maximum values and the theoretical values; this is mainly caused by assuming that the input light is vertical incidence and neglecting the coupling loss between two optical fibers. The surface of the actual F-P cavity is not perfectly vertical and the existence of the coupling loss of two fibers will also lead to the deviations. Figure 4 shows the asymmetric fiber F-P cavity reflectivity curves in the wavelength range of $1545 \mathrm{~nm}-1552 \mathrm{~nm}$ with the good linearity, and the linear fitting coefficient is 0.9978 . So we chose the asymmetric optical fiber F-P cavity whose working range was $1545 \mathrm{~nm}-1552 \mathrm{~nm}$ and wavelength tuning range of the sensing FBG was around $1545 \mathrm{~nm}-1552 \mathrm{~nm}$. This ensures that the sensing wavelength is always in the linear region of the reflection spectrum of the F-P cavity.

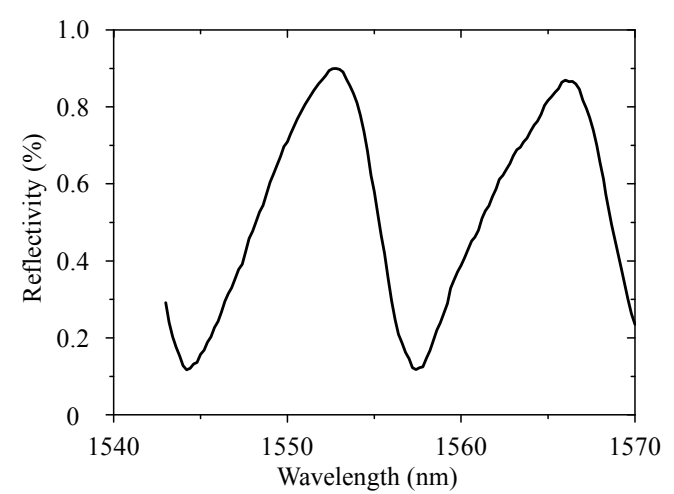

Fig. 4 Reflectivity curve of the asymmetric optical fiber F-P cavity.

An experimental setup for the FBG signal demodulation system based on the edge filter theory was conducted to verify the proposed method. Experiments were performed using the following parameters: the central wavelength of the super light emitting diode (SLED) broadband light source was $1550 \mathrm{~nm}$, the bandwidth was $40 \mathrm{~nm}$, and the total power output was $1 \mathrm{~mW}$. The central wavelength of the sensing FBG was $1549.92 \mathrm{~nm}$; the $3-\mathrm{dB}$ bandwidth was about $0.2 \mathrm{~nm}$, and the peak reflectivity was about $95 \%$. The optical powers of $P_{1}$ and $P_{2}$ were detected by a New-Port $1830-\mathrm{C}$ optical power meter. And the reflected wavelength was monitored by an Anritsu MS9710C spectrum analyzer. The FBG sensor was mounted on a cantilever beam to produce the wavelength shift. Figure 5 shows the reflected spectrum of the sensing probe demodulated by the non-symmetric optical fiber F-P cavity. As shown in Fig. 5, the intensity of the FBG sensor changes with the wavelength shift of the sensing probe's reflection spectrum.

Figure 6 shows the experimental curves of the ratio of $P_{1}$ and $P_{2}$ with the reflection spectrum wavelength of the FBG sensor. It can be seen from Fig. 6 that the signal light and reference light measured power ratio has a good linear relationship with the FBG sensing wavelength, and the linear 
fitting is 0.9973 . The wavelength resolution of the demodulation system is $0.01 \mathrm{~nm}$, and the wavelength demodulation range of the demodulation system is $7 \mathrm{~nm}$. This can be obtained by analyzing the linear filtering range of the asymmetric optical fiber F-P cavity.

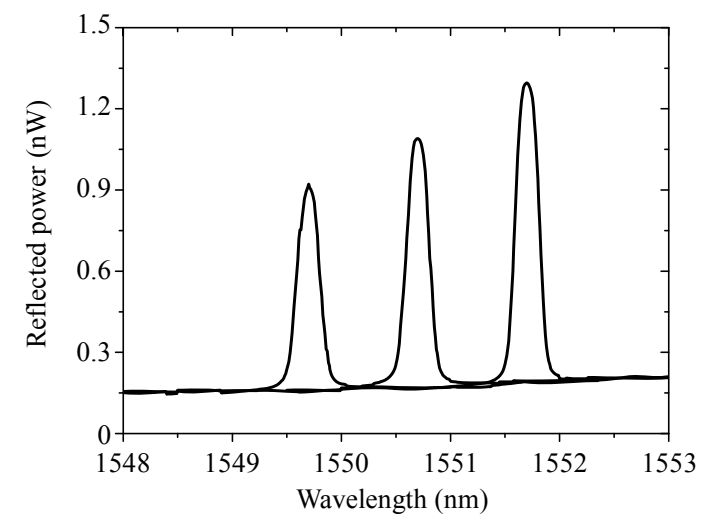

Fig. 5 Reflective spectrum at different wavelength shifts of the FBG.

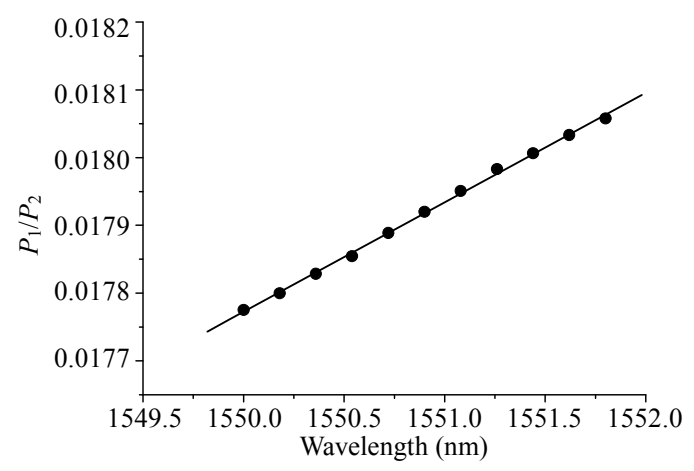

Fig. $6 R(\lambda)$ vs. wavelength shift of the FBG sensor.

\section{Conclusions}

An FBG sensor edge-filter demodulation system based on the tunable asymmetric fiber F-P cavity is reported in this paper. The asymmetric fiber F-P cavity was fixed on two coated fibers with different optical thin films. Based on the theory of optical thin film interference, the tunable asymmetric F-P interference cavity with the wide linear range was designed, and the optimal parameters of the structure were obtained. The asymmetric fiber F-P cavity was used for the linear edge filter to demodulate the wavelength of the FBG sensor. Experimental results show that this FBG wavelength detection system can work efficiently for the measurement of the weak signal. And it gives a wavelength resolution of 0.01 picometer with the linear wavelength shift range of $7 \mathrm{~nm}$. The sensor demodulation system has the advantages of the simple structure, easy adjustment of the filter curve, good linearity, and low requirement of working environment.

Open Access This article is distributed under the terms of the Creative Commons Attribution License which permits any use, distribution, and reproduction in any medium, provided the original author(s) and source are credited.

\section{References}

[1] J. Corres and I. Villar, "Two-layer nanocoatings in long-period fiber gratings for improved sensitivity of humidity sensors," IEEE Transactions on Nanotechnology, 2008, 7(4): 394-400.

[2] J. R. Guzman-Sepulveda and D. A. May-Arrioja, "In-fiber directional coupler for high-sensitivity curvature measurement," Optics Express, 2013, 21(10): 11853-11861.

[3] B. B. Gu, M. J. Yin, A. P. Zhang, J. W. Qian, and S. L. $\mathrm{He}$, "Optical fiber relative humidity sensor based on FBG incorporated thin-core fiber modal interferometer," Optics Express, 2011, 19(5): 4140-4146.

[4] G. Luyckx and E. Voet, "Strain measurements of composite laminates with embedded fiber Bragg gratings: criticism and opportunities for research," Sensors (Basel), 2011, 11(1): 384-408.

[5] S. J. Jiang, Y. C. Liang, X. Zhu, and H. H. Wang, “An asymmetric Fabry-Perot interferometric cavity for fiber optical sensors," Chinese Optics Letters, 2006, 4(10): 563-565.

[6] C. S. Kim and J. U. Kang, "Multi-wavelength switching of Raman fiber ring laser incorporating composite polarization-maintaining fiber LyotSagnac filter," Applied Optics, 2004, 43(15): 31513157.

[7] D. H. Kim and J. U. Kang, "Sagnac loop interferometer based on polarization maintaining photonic crystal fiber with reduced temperature sensitivity," Optics Express, 2004, 12(19): 4490 4495.

[8] Q. Wu, G. Farrell, and Y. Semenova, "Simple design technique for a triangular FBG filter based on a linearly chirped grating," Optics Communications, 2010, 283(6): 985-992. 
[9] A. M. Hattaa, G. Farrell, Y. Semenov, and Q. Wang, "A simple integrated ratiometric wavelength monitor based on a directional coupler," Optik - International Journal for Light and Electron Optics, 2014, 126(2): 795-798.

[10] B. Yang, W. C. Shi, C. Y. Pei, and H. Yu, "Dual Mach-Zehnder based integrated X-type ratiometric wavelength monitor on glass," IEEE Photonic
Technology Letters, 2014, 26(5): 433-435.

[11] K. Toge and F. Ito, "Recent research and development of optical fiber monitoring in communication systems," Photonic Sensors, 2013, 3(4): 304-313.

[12] K. K. Chin and Y. Sun, "Fabry-Perot diaphragm fiber-optic sensor," Applied Optics, 2007, 46(31): 7614-7619. 